\title{
Low complexity QoS-aware subcarrier allocation for MISO-OFDMA systems
}

\author{
Mahmoud M. Selim ${ }^{1,3 a)}$, Osamu Muta ${ }^{2}$, Hossam M. H. Shalaby ${ }^{1}$, \\ and Hiroshi Furukawa ${ }^{3}$ \\ ${ }^{1}$ Egypt-Japan University of Science and Technology (E-JUST), \\ New Borg Al-Arab, Alexandria, Egypt \\ ${ }^{2}$ Center for Japan-Egypt Cooperation in Science and Technology, \\ Kyushu University, Fukuoka-shi, Fukuoka, Japan \\ ${ }^{3}$ Graduate School of Information Science and Electrical Engineering, \\ Kyushu University
}

a)mahmoud.sleem@ejust.edu.eg

\begin{abstract}
A low complexity Quality-of-Service (QoS)-aware sub-carrier allocation/user-selection technique for the downlink of Multiple-Input Single-Output Orthogonal Frequency Division Multiple-Access (MISOOFDMA) system is proposed. The algorithm allows simultaneous transmission to multiple users on the same sub-carrier through zero-forcing beamforming (ZFBF). The proposed algorithm enhances QoS by preserving minimum rate for each user while providing high bandwidth data services. Simulation results reveals that our algorithm outperforms other reference algorithms in terms of average sum-rate per sub-carrier and QoS, while lower complexity is attained by the proposed algorithm in terms of execution time needed compared to reference algorithms.
\end{abstract}

Keywords: sub-carrier allocation, MISO, OFDMA, ZFBF, fairness, QoS Classification: Wireless Communication Technologies

\section{References}

[1] 3GPP, Requirements for further advancements for E-UTRA (LTE-Advanced), 3rd Generation Partnership Project (3GPP), TR 36.913, Jun. 2008.

[2] Q. H. Spencer, A. L. Swindlehurst, and M. Haardt, "Zero-forcing methods for downlink spatial multiplexing in multiuser MIMO channels," IEEE Trans. Signal Process., vol. 52, no. 2, pp. 461-471, Feb. 2004. DOI:10.1109/TSP.2003.821107

[3] M. Costa, "Writing on dirty paper," IEEE Trans. Inf. Theory, vol. 29, no. 3, pp. 439-441, May 1983. DOI:10.1109/TIT.1983.1056659

[4] V. Papoutsis and S. Kotsopoulos, "Resource allocation algorithm for MISOOFDMA systems with QoS provisioning," Proc. $7^{\text {th }}$ ICWMC 2011, International Conference on Wireless and Mobile Communications, Luxembourg, Luxembourg, pp. 7-11, June 2011.

[5] I. Basturk and B. Ozbek, "Fairness aware resource allocation for downlink MISOOFDMA systems," Proc. $13^{\text {th }}$ Wireless Communications and Networking Conference (WCNC), 2012 IEEE, Paris, France, pp. 188-192, April 2012. DOI:10.1109/ WCNC.2012.6214094 
[6] A. J. Goldsmith and S.-G. Chua, "Variable-rate variable-power M-QAM for fading channels,” IEEE Trans. Commun., vol. 45, no. 10, pp. 1218-1230, Oct. 1997. DOI: $10.1109 / 26.634685$

\section{Introduction}

Orthogonal Frequency Division Multiple-Access (OFDMA) is a premier technology in fighting multipath fading and supporting high data rate services in modern wireless systems [1]. Combining Multi-Input Multi-Output (MIMO) technology with OFDMA increases system capacity, but complicates allocating resources (subcarriers, power and bit loading) efficiently among users.

Simultaneous transmission from base station (BS) with $T$ transmit antennas to multiple users on the same sub-carrier increases system throughput, but additional complexity is required for mitigating inter-user interference and selecting users on each sub-carrier. In literature, sub-optimal solutions based on linear precoding techniques such as zero-forcing beamforming (ZFBF) [2] have been proposed for inter-user interference cancellation based on linear filtering due to its simple implementation compared to theoretical optimal solution of dirty paper coding (DPC) which is practically very difficult to implement [3]. Also, different user selection algorithms based on ZFBF have been proposed in literature [4, 5]. The algorithm in [4] maximizes system capacity with Quality-of-Service (QoS) provision in a two-step resource allocation that preserves minimum user rate for each user, while the algorithm in [5] also maximizes system capacity but selects users based on proportional rates achieved to guarantee fairness among users.

The original contribution of this paper is to propose a low complexity QoSaware one-step resource allocation algorithm for downlink transmission of MultiInput Single-Output OFDMA (MISO-OFDMA) system. The proposed algorithm cancels interference among users through ZFBF and efficiently selects users on each sub-carrier to maximize system sum rate under minimum user rate constraint preserved for each user so as to enhance QoS like algorithm in [4] but with limited complexity.

\section{System model and problem formulation}

We consider a single-cell OFDMA system with $N$ sub-carriers served by one BS equipped with $T$ transmit antennas and single-antenna $K$ users are uniformly distributed such that $K \geq T$. To allow transmission to multiple users on the same sub-carrier $n$, transmit beamforming is required and the received component by any user $k \in\{1,2, \ldots, K\}$ on sub-carrier $n, y_{k, n}$, is given by

$$
y_{k, n}=\mathbf{h}_{k, n} \mathbf{w}_{k, n} \sqrt{p_{k, n}} s_{k, n}+\sum_{\substack{j=1 \\ j \neq k}}^{K} \mathbf{h}_{k, n} \mathbf{w}_{j, n} \sqrt{p_{j, n}} s_{j, n}+z_{k, n}
$$

where for any user $k$ on sub-carrier $n, \mathbf{h}_{k, n}=\left[h_{k, n}^{1} h_{k, n}^{2} \ldots h_{k, n}^{T}\right]$ is the complex channel gain vector, $\mathbf{w}_{k, n}=\left[w_{k, n}^{1} w_{k, n}^{2} \ldots w_{k, n}^{T}\right]^{t}$ is the corresponding beamforming vector, $p_{k, n}$ is the power transmitted (homogeneous power allocation among sub- 
carriers and water-filling among users on same sub-carrier are assumed as in [4, 5]), $s_{k, n}$ is transmitted OFDM symbol and $z_{k, n}$ is circular symmetric complex Gaussian noise with zero mean and variance $\sigma_{\eta}^{2}$. The first and second terms in (1) denote signal desired component and signal inter-user interference component, respectively.

As $T<K$, we need to select a set of users $A \subset\{1,2, \ldots, K\}$ and $0<|A| \leq T$ on each sub-carrier, where |.| denotes set cardinality. To cancel interference among users of set $A$ assigned on sub-carrier $n$ (second term in (1)), we employ ZFBF [2] where the beamforming matrix $\tilde{\mathbf{W}}_{n}(A)=\left[\tilde{\mathbf{w}}_{1, n} \ldots \tilde{\mathbf{w}}_{|A|, n}\right]$ is given by

$$
\tilde{\mathbf{W}}_{n}(A)=\mathbf{H}_{n}(A)^{H}\left(\mathbf{H}_{n}(A) \mathbf{H}_{n}(A)^{H}\right)^{-1} .
$$

Here, $\mathbf{H}_{n}(A)=\left[\mathbf{h}_{1, n}^{t} \ldots \mathbf{h}_{|A|, n}^{t}\right]^{t}$ is channel matrix of set $A$ on sub-carrier $n$. For power normalization, pre-coding vector for user $k \in A$ on sub-carrier $n$ is given by

$$
\mathbf{w}_{k, n}=\sqrt{\frac{|A|}{\operatorname{tr}\left(\tilde{\mathbf{W}}_{n}(A) \tilde{\mathbf{W}}_{n}(A)^{H}\right)}} \tilde{\mathbf{w}}_{k, n}
$$

The data rate achieved by user $k \in A$ on sub-carrier $n$ is given by

$$
r_{k, n}=\log _{2}\left(1+p_{k, n} \gamma_{k, n}\right)
$$

where $\gamma_{k, n}=\frac{\left|\mathbf{h}_{k, n} \mathbf{w}_{k, n}\right|^{2}}{\sigma_{n}^{2}}$ and $\Gamma=-\frac{\ln \left(5 B E R_{k, n}\right)}{1.5}$. BER $R_{k, n}$ is bit error rate encountered on the $n^{\text {th }}$ sub-carrier of the $k^{\text {th }}$ user and is approximately formulated for $l$-ary QAM modulation as [6]

$$
B E R_{k, n} \approx 0.2 \exp \left(\frac{c \cdot \gamma_{k, n}}{l_{k, n}-1}\right)
$$

where $c=-1.6$ and $l_{k, n}$ is the highest $l$-ary QAM modulation on sub-carrier $n$ that makes $B E R_{k, n} \leq B E R_{t h}$. Total rate achieved by user $k$ is given by

$$
R_{k}=\sum_{n=1}^{N} \rho_{k, n} \cdot r_{k, n}
$$

where $\rho_{k, n} \in\{0,1\}$ is a binary variable to determine weather sub-carrier $n$ is assigned to user $k\left(\rho_{k, n}=1\right)$ or not $\left(\rho_{k, n}=0\right)$.

\section{Proposed resource allocation algorithm}

Our proposed sub-carrier-wise resource allocation algorithm is described in details in Algorithm 1. The proposed algorithm provides a sub-optimal solution for subcarrier allocation and user selection dual problem with low complexity and QoS provision.

In sub-carrier allocation step, to enhance QoS, at each sub-carrier $n \in$ $\{1,2, \ldots, N\}$, user $k_{\text {opt }}$, with the highest channel gain vector norm on the $n^{\text {th }}$ sub-carrier, is chosen among a set $\psi_{0}$ of users who meet $R_{k}<R_{t h}$, where $R_{t h}$ denotes the target minimum user rate set as a QoS constraint. If users set, $\psi_{0}$, becomes empty, user $k_{\text {opt }}$ is chosen among set of all users $\psi$ instead and assigned to sub-carrier $n$. After achievable rate by user $k_{\text {opt }}$ on sub-carrier $n$ is calculated according to (4), channel matrix $\mathbf{H}_{n}(A)$ is updated and the remaining users with small rates $\left(R_{k}<R_{t h}\right)$ are considered as candidates for further assignment on the same sub-carrier in next user selection step. 


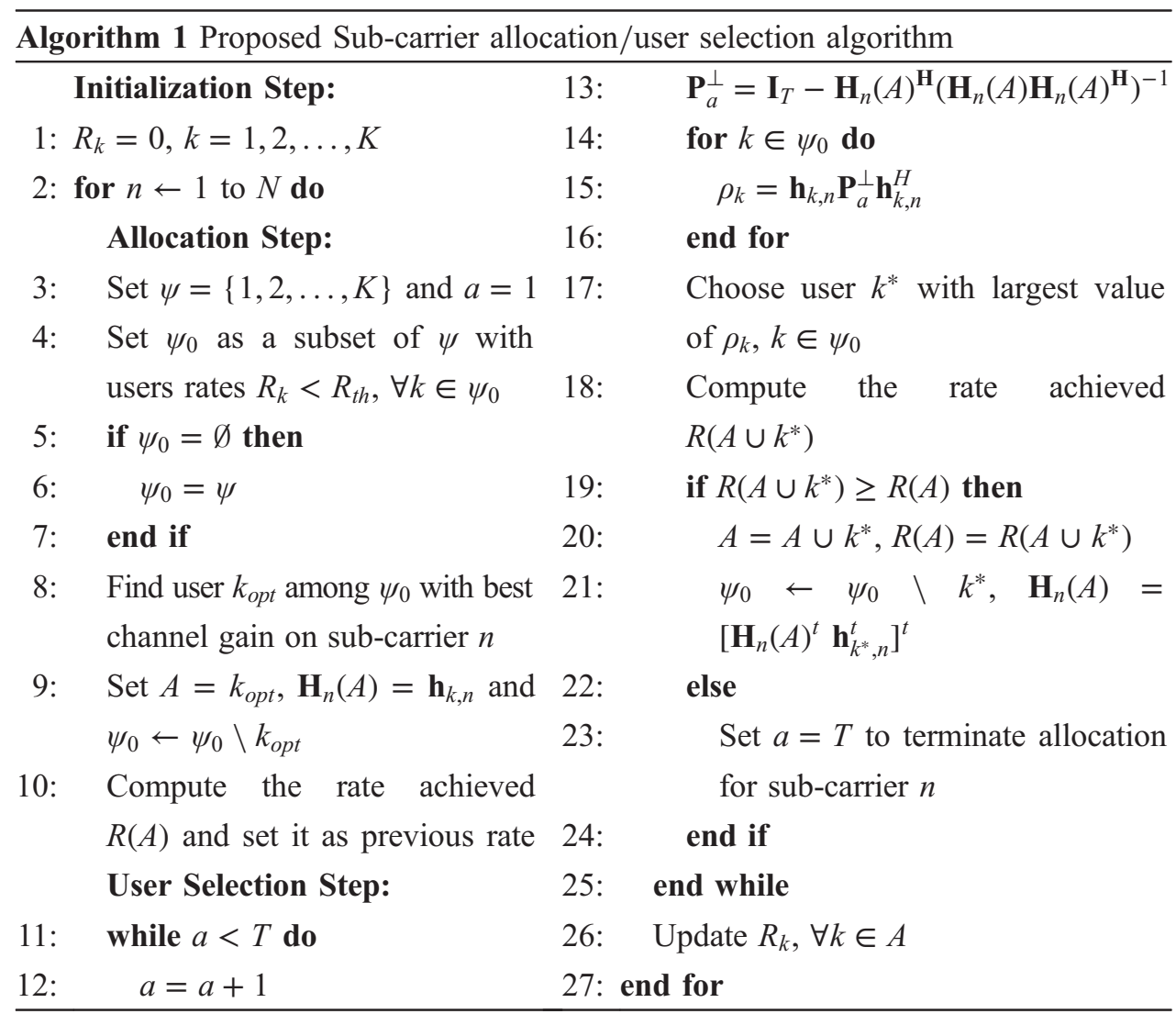

In user selection step, additional users on the same sub-carrier $n$ are added one by one with maximum $T$ users (controlled by parameter $a$ ). User selection is performed by calculating the projection of each candidate user, $\rho_{k}, \forall k \in \psi_{0}$ on the orthogonal complement of subspace spanned by assigned user(s) so far, denoted as $\mathbf{P}_{a}^{\perp}$, and user $k^{*} \in \psi_{0}$ with the highest value of $\rho_{k}, \forall k \in \psi_{0}$, is chosen. If commutative achievable rate on sub-carrier $n$ is increased by adding user $k^{*}$, sub-carrier $n$ is assigned to user $k^{*}$. After that, channel matrix $\mathbf{H}_{n}(A)$ is updated and selection step continues for further addition of users. Otherwise, selection step is terminated and rates of users selected on sub-carrier $n$ are updated. The same procedure is carried out for all available sub-carriers.

\section{Simulation \& results}

We simulate downlink transmission of single-cell MISO-OFDMA system with $T=4$ and $N=64$, where we assume Rayleigh-fading channel model with 6 taps, exponential power decay profile (PDP) and maximum delay spread of $5 \mu \mathrm{s}$ with OFDM symbol duration of $64 \mu \mathrm{s}$. BER constraint is $B E R_{t h}=10^{-3}$ and minimum user rate per sub-carrier is $\frac{R_{t h}}{N}=1.5 \mathrm{bps} / \mathrm{Hz}$ as in [4]. Perfect channel state information (CSI) is assumed at the transmitter.

Fig. 1(a) shows average sum rate per sub-carrier against signal to noise power ratio (SNR) in $\mathrm{dB}$ for $K=10$ users for our proposed algorithm, two reference algorithms in [4,5] and Round Robin (RR) algorithm that selects users regardless of channel conditions. Our proposed algorithm outperforms other reference algorithms because it avoids re-allocating sub-carriers step encountered in algorithm [4] 


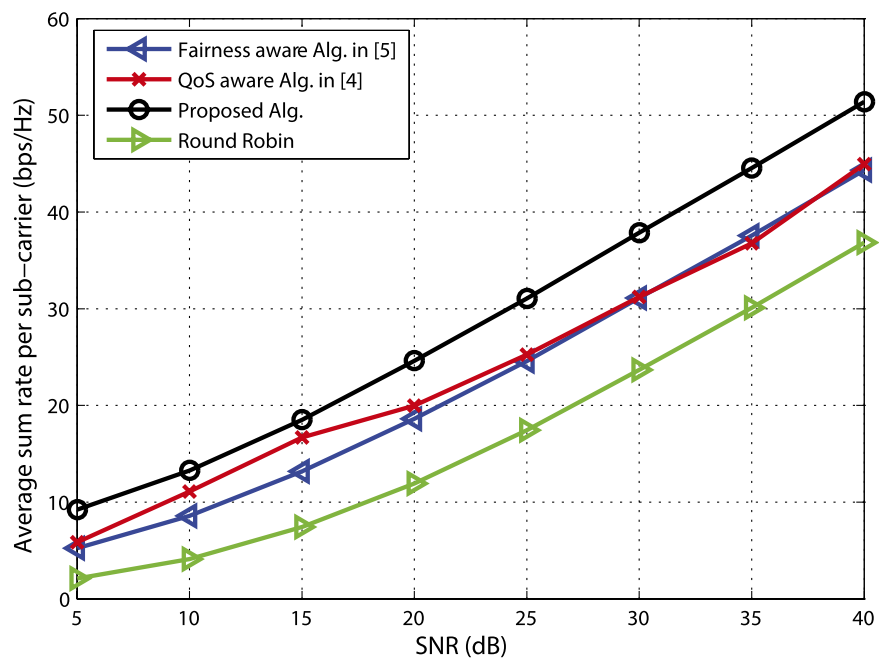

(a) Average sum rate per sub-carrier vs. SNR.

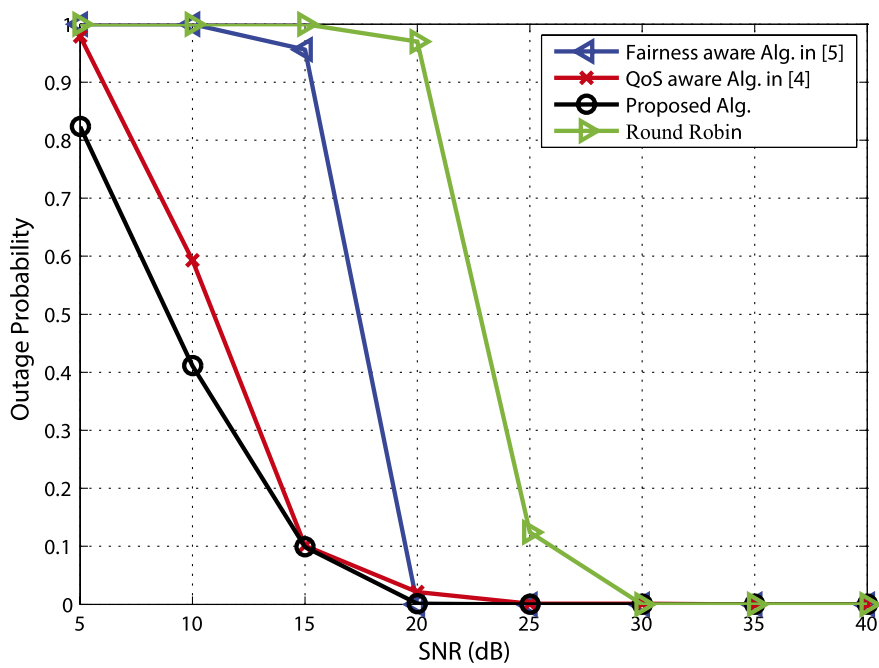

(b) Outage Probability vs. SNR.

Fig. 1. Performance comparison in terms of average sum-rate and outage probability.

that may degrade performance of some users so as to enhance total QoS and also avoids fairness exaggeration encountered in algorithm [5] that may hinder efficient sub-carrier allocation to guarantee fairness.

Fig. 1(b) shows outage probability defined as percentage of users failed to achieve predefined minimum user rate per sub-carrier against SNR for $K=10$ users. The results show that our proposed algorithm outperforms QoS provision approach in [4] by avoiding re-allocation step that may result in performance degradation for some users especially for low SNR transmission. Our proposed algorithm also outperforms fairness-aware approach in [5] as this approach tends to achieve almost equal rates for different users in the cell which requires high SNR transmission to make these rates exceed the minimum target user rate as shown in Fig. 1(b).

We define complexity as average execution time needed by different algorithms to perform the whole allocation process until all sub-carriers are assigned to users. This execution time is calculated by "TIC" MATLAB function that starts stopwatch 
Table I. Average execution time (msec) of different algorithms with variable number of users $K$ at $\mathrm{SNR}=20 \mathrm{~dB}$.

\begin{tabular}{c|c|c|c|c}
\hline Number of users & Algorithm in [5] & Algorithm in [4] & Proposed & RR \\
\hline $\mathrm{K}=6$ & 157.4 & 185.7 & 101.2 & 9.5 \\
\hline $\mathrm{K}=8$ & 176.1 & 503.5 & 110.3 & 9.3 \\
\hline $\mathrm{K}=10$ & 187.2 & 1289.9 & 118.4 & 9.3 \\
\hline $\mathrm{K}=12$ & 197.5 & 2517.8 & 125.3 & 9.3 \\
\hline $\mathrm{K}=14$ & 208 & 3744 & 131.1 & 9.3 \\
\hline $\mathrm{K}=16$ & 218.5 & 5425.9 & 136.6 & 9.3 \\
\hline
\end{tabular}

timer and "TOC" MATLAB function that calculates time elapsed since "TIC" was started, where a 32-bit machine with Intel(R) Core(TM)2 Due at $2.00 \mathrm{GHz}$ processor and $4.00 \mathrm{~GB}$ RAM are used. Table I shows average execution time needed by different algorithms for different number of active users $K$ deployed in the cell at average SNR $=20 \mathrm{~dB}$. As expected, Round Robin is the simplest allocation methodology which attains the lowest execution time but with lowest throughput. As algorithm [4] performs resource allocation in a two-step process, it requires very huge execution time that highly grows with number of users as shown in Table I. The results in Table I also show that our proposed algorithm halved the execution time needed compared to fairness aware algorithm in [5] by avoiding calculations needed for user selection based on proportional rates. This clearly reveals that the proposed algorithm achieves the lowest complexity except for RR.

\section{Conclusion}

A sub-carrier allocation/user selection algorithm for the downlink of MISOOFDMA system based on ZFBF has been proposed. Simulation results have clarified that the proposed algorithm outperforms three reference algorithms in terms of average sum rate per sub-carrier, while outage probability (measurement of QoS) is decreased especially for low SNR values by considering minimum user rate for each user during allocation. Complexity has been measured through execution time needed and the results reveal that the proposed algorithm attains lower complexity than the reference ones. 